\title{
Open-Access Microcavities for Chemical Sensing
}

\author{
Claire Vallance $^{a}$, Aurelien A. P. Trichet ${ }^{b}$, Dean James ${ }^{a}$, Philip R. Dolan ${ }^{b}$, and Jason M. Smith ${ }^{b}$ \\ ${ }^{a}$ Department of Chemistry, University of Oxford, Chemistry Research Laboratory, 12 Mansfield Rd, Oxford \\ OX1 3TA, UK; 'bepartment of Materials, University of Oxford, 16 Parks Rd, Oxford OX1 3PH
}

\begin{abstract}
The recent development of open-access optical microcavities opens up a number of intriguing possibilities in the realm of chemical sensing. We provide an overview of the different possible sensing modalities, with examples of refractive index sensing, optical absorption measurements, and optical tracking and trapping of nanoparticles. The extremely small mode volumes within an optical microcavity allow very small numbers of molecules to be probed: our current best detection limits for refractive index and absorption sensing are around $10^{5}$ and $10^{2}$ molecules, respectively, with scope for further improvements in the future.
\end{abstract}

\section{Introduction}

Cavity-enhanced absorption spectroscopy is a proven method for ultra-sensitive detection of a range of chemical species, and has been used widely for chemical sensing applications in areas ranging from atmospheric chemistry and environmental and process monitoring to plasma diagnostics and breath analysis ${ }^{1}$. The method owes its extraordinary sensitivity to the exceptionally long absorption path lengths that can be achieved when the excitation light is confined within an optical cavity, within which the light undergoes many thousands of passes through the sample. In gas-phase applications, limits of detection in the parts-per-billion to parts-per-trillion range are routinely reached, and the technology has reached a level of maturity such that commercial trace gas analysers based on cavity-enhanced spectroscopy are available for a number of small-molecule analytes ${ }^{2,3}$. Despite the widespread popularity of cavity-enhanced detection methods for gas-phase measurements, there have been relatively few reported applications in the liquid or solution phase. This is due in part to the fact that the much higher molecular densities in the condensed phase mean that there are fewer liquid-phase applications in which such high detection sensitivities are required, but also due to the considerable challenges associated with maintaining a high-finesse optical cavity following the introduction of a liquid sample. Cavity-enhanced absorption measurements become an appealing option when extremely small volumes of liquid must be probed and/or the single-pass path length through the sample is very small. This may be due to the availability of a limited amount of sample, often the case for biological samples, for example. A key potential application area lies in 'lab-on-a-chip' devices, also known as micro total analysis systems ( $\mu$ TAS). Intrinsic path lengths across a microfluidic channel are often in the range from 50 to $100 \mu \mathrm{m}$, and enhancing this path length by encapsulating the channel within an optical cavity offers the potential for dramatic improvements in detection sensitivity. There are two possible approaches: either the device may be placed within a conventional optical cavity, usually consisting of a pair of carefully aligned, highly-reflective mirrors; or the cavity may be incorporated into the device itself. We have demonstrated the former 'device in a cavity' approach previously ${ }^{4,5,6,7}$. However, fabricating devices with optical properties compatible with cavity-enhanced measurements, and achieving optimum (or even adequate) overlap between the spatial modes of the cavity and the microfluidic channel without suffering significant scattering losses, is extremely challenging. The second approach of using a miniature cavity is thus attractive if sufficiently high quality cavities can be fabricated. Miniaturisation of the cavity modes brings additional advantages for sensing modalities that involve spontaneous emission or scattering, since the small optical mode volume leads to quantum-mechanical effects that are strong enough to be used to enhance detection sensitivity. A number of authors have previously reported sensing applications of whisperinggallery-mode microcavities ${ }^{8,9}$, demonstrating high detection sensitivities based on measureable shifts in cavity mode frequencies induced by binding of a target molecule to a functionalised surface. Open-access optical microcavities, which have been produced by a number of methods over the past ten years ${ }^{10,11,12,13,14,15,16}$, offer an alternative 'cavity in a device' approach, in which the optical field can be confined to dimensions similar to the wavelength of the resonant light employed. In particular, the use of 
focused-ion-beam milling to fabricate mirror substrates allows precision engineering of the cavity shape, and the realisation of arrays of cavities for highly parallel chip-scale sensors. To date, there are only one or two examples of sensing using open microcavities ${ }^{17,18,19}$. However, we believe that open-access optical microcavities offer a number of advantages over WGM sensors. They offer the option of bulk measurements in addition to surface-sensitive measurements, as well as being reusable, easily tuneable in wavelength/frequency, and potentially mass-producible, providing a generic platform for a variety of sensing methods.

In the following, we will provide a brief overview of the theory of optical cavities as applied to open-access microcavities, describe the use of optical microcavities in refractive index sensing, absorption sensing, and nanoparticle tracking and trapping, and consider possible future developments in chemical and biochemical applications of optical microcavity sensors. For simplicity, and to highlight the results achieved so far, we limit our discussion to a classical consideration of light confinement in a microcavity and the strength of interaction with intracavity media.

\section{Theory of optical microcavities}

An optical cavity may be thought of as any structure capable of trapping light in the form of a standing wave or cavity mode. The optical microcavities to be employed in the present work are plano-concave Fabry-Pérot cavities comprised of a pair of highly reflective mirrors, aligned such that light entering the cavity is repeatedly reflected back and forth between the mirrors. To undergo constructive interference and form a standing wave within the cavity, a half integer number of wavelengths, $\lambda$, must match the cavity length, $d$. The resonant wavelengths form a series of standing waves within the cavity known as longitudinal modes, satisfying the condition

$$
\frac{m \lambda}{n}=2 d
$$

where $n$ is the refractive index of the medium within the cavity, and $m$ is an integer known as the mode number. Light propagating slightly off-axis can also excite a series of transverse modes within the cavity. All of the cavity modes are solutions of Maxwell's equations subject to the refractive index structure imposed by the mirrors.

Most Fabry-Perot cavities used for sensing applications have lengths of centimetres or even metres, with longitudinal mode spacings (free spectral ranges), $\Delta v=c /(2 n d)$, so small that the modes are often treated as spectrally continuous. In contrast, the free spectral range of a microcavity is so large that only one or a few cavity modes fall within the reflectivity bandwidth of the mirrors. Individual cavity mode frequencies can be tracked as a means of monitoring changes in cavity length or the refractive index of the cavity contents, and mode intensities provide a hallmark of wavelength-dependent optical absorption. We note that for cavities with micron-sized dimensions, the penetration depth of the excitation light into the cavity mirrors can make a significant contribution to the effective cavity length (i.e. the optical path length per pass), and this must be taken into account fully when analysing data from microcavity measurements. We return to this point in more detail in Section 4.1.

The intensity of light trapped in a cavity mode gradually decays away due to losses associated with imperfect reflectivity of the mirrors, and with scattering or absorption at the mirrors or by the medium within the cavity. The lifetime, $\tau$, of the mode is known as the ringdown time. Assuming that both the intrinsic cavity losses and losses associated with the sample are low, the ringdown time depends on the length of the cavity, $d$, the intrinsic fractional loss $L$ within the cavity in the absence of a sample, and the loss per pass, $\alpha C l$ due to absorption by the sample (with $\alpha$ the sample absorption coefficient, $C$ the concentration, and $\ell$ the singlepass absorption path length).

$$
\tau=\frac{n d}{c(L+\alpha C \ell)}
$$

The ringdown time of a microcavity is typically less than $1 \mathrm{~ns}$, too short for direct measurement to be a sensible means of determining the sample absorption, but we may instead exploit the fact that under our 
experimental conditions, the total intensity of light transmitted through the cavity is proportional to the ringdown time. A calibration measurement is required in order to determine the intrinsic cavity losses, $L$. When the sample absorption is much smaller than the intrinsic losses, the absorption of the sample per unit path length, $\alpha C$, can be determined from a measurement of the transmitted light intensity, $I$, in the presence and absence $\left(I_{0}\right)$ of the sample ${ }^{20}$.

$$
\alpha C=\frac{1}{\ell}\left(\frac{I_{0}-I}{I}\right) L
$$

This approach is known as cavity-enhanced absorption spectroscopy (CEAS). Note that the smaller the intrinsic cavity losses, the smaller (i.e. better) the limit of detection for absorption measurements.

The quantity $L^{-1}$ is known as the cavity enhancement factor, and represents the increase in sensitivity of a cavity-based measurement relative to a single-pass measurement. The cavity enhancement factor can be related to two figures of merit for the cavity quality, namely the quality factor (or Q-factor) and the cavity finesse. The quality factor, $Q$, is proportional to the number of optical cycles before the mode intensity decays to $1 / e$ of its initial value, while the finesse, $F$, is proportional to the number of round trips of the cavity before the mode intensity decays to $1 / e$ of its initial value. The two parameters may also be expressed in terms of the mode frequency, $v$, the mode width, $\delta v$, and the cavity free spectral range, $\Delta v$.

$$
\begin{aligned}
& Q=2 \pi\left(\frac{\text { Energy stored }}{\text { Energy dissipated per optical cycle }}\right)=\frac{v}{\delta v} \\
& F=2 \pi\left(\frac{\text { Energy stored }}{\text { Energy dissipated per round trip }}\right)=\frac{\Delta v}{\delta v}=Q \frac{\Delta v}{v}
\end{aligned}
$$

Optimising the sensitivity in any cavity-enhanced sensor relies on maximising these quantities, which is achieved by minimising the intrinsic cavity loss, $L$. In practice, this means maximising the optical quality of the mirror substrates and the mirror reflectivity at the wavelength(s) of interest, and minimising losses associated with any further surfaces or sources of scattering within the cavity.

\section{Microcavity and sensor fabrication}

The technology for fabricating optical microcavity arrays has been developed in Oxford over the past five years $^{10,11}$. Arrays of concave microcavity mirror substrates, with radii of curvature as small as $2 \mu \mathrm{m}$ and surface roughness below $1 \mathrm{~nm}$, are fabricated using focused ion beam (FIB) milling, and are then mirrorcoated with dielectric $\mathrm{Bragg}$ reflectors consisting of alternating layers of $\mathrm{SiO}_{2}$ and $\mathrm{TiO}_{2}$ to achieve the required reflectivity in the wavelength region of interest. An array of concave features on a substrate prepared in this way is shown in Figure 1(a).

An array of plano-concave microcavities is then formed by coupling a micro-mirror array with a planar mirror (see Figure 1(b)). One or both mirrors are generally mounted on piezoelectric stages in order to allow precise control over the cavity length and relative angular alignment of the mirrors. When the mirror separation is less than the radius of curvature of the concave mirrors, the cavity modes are stable, and are well confined in all three dimensions. With spherical concave mirrors, the confined modes take approximately HermiteGauss or Laguerre-Gauss forms. A typical longitudinal $\left(T E M_{00}\right)$ cavity mode is shown in Figure $1(b)$. The free spectral range of the microcavities is such that only one or a few cavity modes are resonant within the cavity over the reflectivity bandwidth of the mirrors, and piezoelectric tuning of the cavity length provides a simple mechanism for tuning the cavity resonances to the wavelengths of interest for the target species. A cavity transmission spectrum for a cavity in which the free spectra range exceeds the mirror reflectivity bandwidth is shown in Figure 1(c), and consists of a single sharp resonance. Typical mode volumes within the cavities are less than one $\mu \mathrm{m}^{3}$. Cavity $Q$ factors up to around $10^{5}$ have been achieved so far, and $Q$ factors up to $10^{6}$ should be realisable with improved surface processing and coating. 


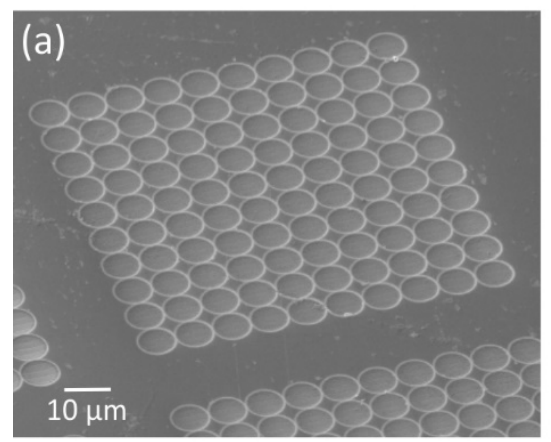

(b)

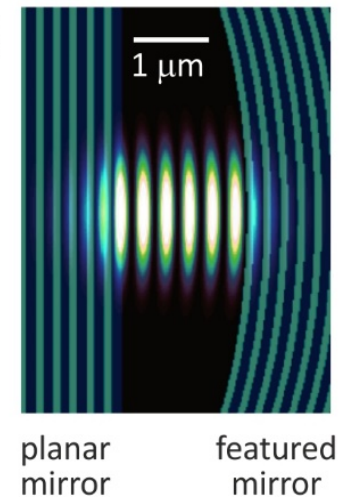

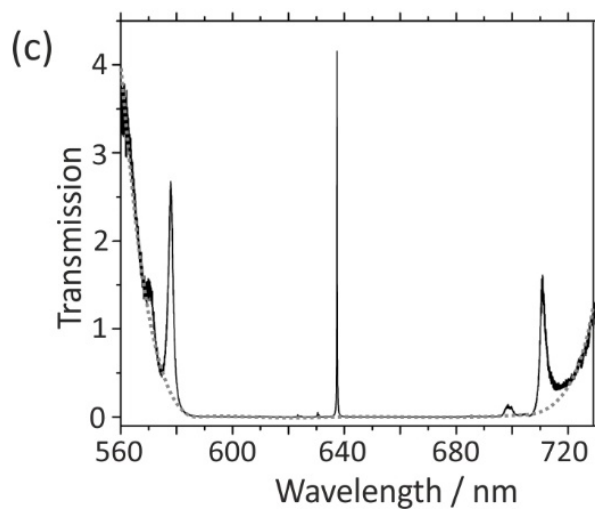

Figure 1: (a) Arrays of $8 \mu \mathrm{m}$ diameter microcavity mirrors; (b) a longitudinal mode within a typical microcavity; (c) cavity transmission spectrum, with the transmission curve of the cavity mirrors superimposed (dotted grey line). Outside the reflectivity bandwidth of the mirrors, light is transmitted directly through the cavity without forming resonant modes. In this case a single longitudinal mode is resonant within the mirror reflectivity bandwidth at $\sim 640 \mathrm{~nm}$, and two broader less well-confined modes are visible at the edges of the reflectivity bandwidth, at $\sim 580$ and $\sim 715 \mathrm{~nm}$, respectively

For chemical sensing measurements, the cavity array is incorporated into a flow cell device, allowing facile introduction of target chemical species into the cavities. An exploded view of a flow cell used for the experiments described in Section 4 is shown in Figure 2. For the refractive index and absorption sensing measurements reported in Sections 4.1 and 4.2, the patterned cavity mirrors have a diameter of $10 \mu \mathrm{m}$, with a $25 \mu \mathrm{m}$ radius of curvature, and comprise ten layers of $\mathrm{SiO}_{2} / \mathrm{TiO}_{2}$ with layer thicknesses tuned to achieve maximum reflectivity of around 0.997 at a wavelength of $640 \mathrm{~nm}$. The resulting cavities have a finesse of around $10^{3}$. For the trapping measurements described in Section 4.3 , the patterned mirrors have a $4 \mu \mathrm{m}$ radius of curvature, and comprise 16 layers of $\mathrm{SiO}_{2} / \mathrm{TiO}_{2}$, with layer thicknesses tuned to achieve absorptionlimited reflectivity (absorption $=20 \mathrm{ppm}$ ) at a wavelength of $640 \mathrm{~nm}$. The resulting cavity modes have a finesse of around 4000. Excitation light from a lamp or laser source is introduced through one of the cavity mirrors, and resonant light transmitted through the cavity is collected by a microscope objective before being directed either to an avalanche photodiode for single wavelength measurements, or a spectrograph for broadband measurements. As we shall see in the following, the flow cell arrangement provides a general platform for chemical sensing via a variety of different sensing modalities.

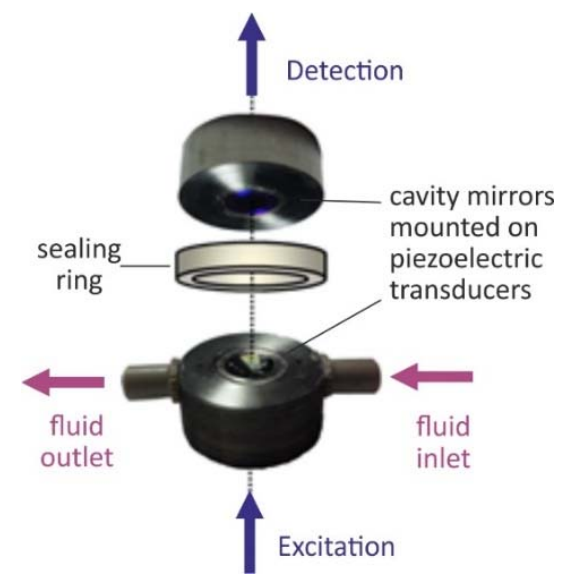

Figure 2: Exploded view of the microcavity flow cell arrangement used for the measurements described in Section 4.

\subsection{Refractive index sensing}


Refractive index measurements are widely used for characterising fluids in industrial, chemical, and food processing applications, as well as to detect contamination events in environmental applications. A change in refractive index of the fluid contained within a microcavity alters the optical path length, leading to a frequency shift in the resonant cavity modes. Using the flow cell setup described above and shown in Figure 2 , we have investigated the sensitivity of the microcavity mode frequencies to refractive index by alternately flowing deionized water and solutions of glucose $\left(1.39 \mathrm{~mol} \mathrm{dm}^{-3}\right)$ through the microcavity array ${ }^{17}$. Glucose was chosen for these measurements due to its very well characterised refractive index. The microcavity array was illuminated with white light from a quartz halogen lamp (Bentham WLS100), and the cavity output spectra were recorded using a spectrograph (Andor, 300 lines per mm grating, multitracked to record spectra simultaneously from 10 cavities every $100 \mathrm{~ms}$ ). A typical data set for these experiments, showing the transmission spectrum of a single microcavity as a function of time as the different solutions are flowed through the cavity, is presented in Figure 3(a). In practice, to determine accurate changes in refractive index, it was necessary to monitor the wavelength shifts across several cavities of slightly different cavity lengths. By monitoring multiple cavities we are able to perform a relative rather than an absolute measurement, such that any noise affecting all cavities (eg. vibrational noise) is removed from our data. Firstly, this allowed differentiation between wavelength shifts caused by refractive index changes and those caused by physical changes in the cavity length as a result of vibrations or experimental drift. Secondly, it allowed the penetration depth of the excitation light into the surface of the cavity mirrors to be determined and corrected for. Penetration of the cavity mode into the cavity mirrors contributes significantly to the optical path length of the mode, but represents a region in which the light is not interacting with the sample.

Monitoring of multiple cavities with slightly different lengths was achieved by employing a wedged geometry, in which the planar mirror was tilted at a slight angle $\left(\sim 0.3^{\circ}\right)$ to the patterned mirror array, such that adjacent cavities had slightly different mirror separations. The data analysis procedures employed to extract the penetration depth and change in refractive index from the resulting data set are described in detail in reference 17, and allow the refractive index within the flowing solution to be determined as a function of time, as shown in Figure 3(b). The observed 'shoulder' on the refractive index signal as the glucose solution flows into the microcavities is thought to be a result of non-optimal flow cell design, such that solutions enter the microcavity arrays via a mixture of flow and diffusion behaviour. The flow dynamics will be improved in future designs of the flow cell.

Using this approach we have demonstrated a sensitivity to changes in refractive index as small as $3.5 \times 10^{-4}$ RIU, corresponding to the detection of around 500,000 glucose molecules within the $\sim 50 \mathrm{fL}$ volume of the monitored microcavities. With improved mirror coatings and a more intense light source, it should be possible to improve considerably on this detection limit in future measurements ${ }^{21}$.

(a)

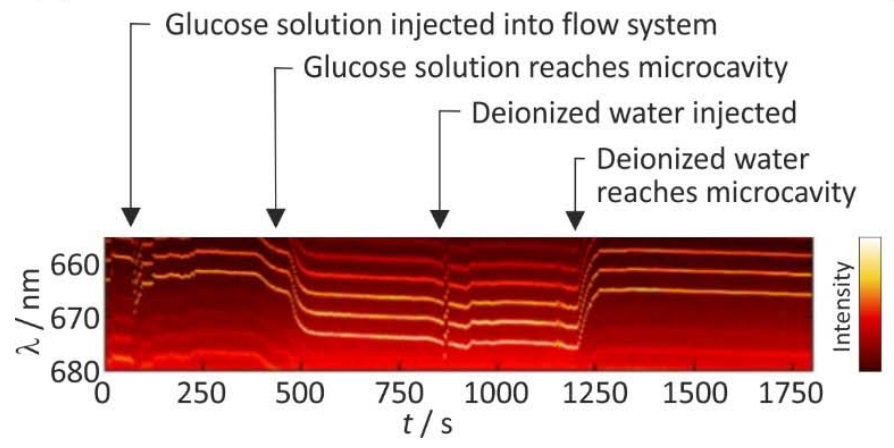

(b)

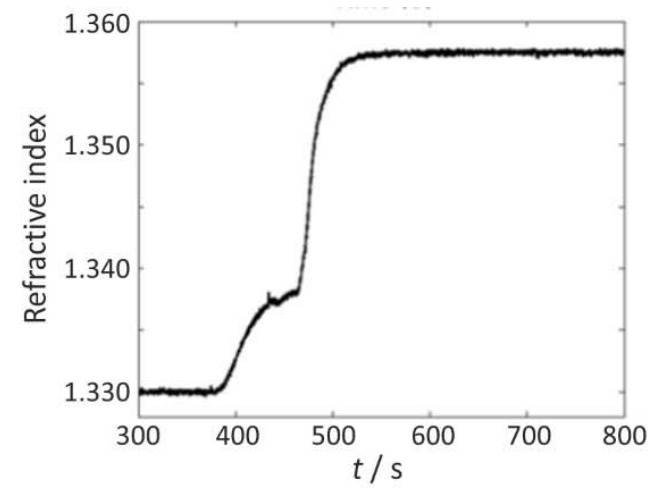

Figure 3: (a) Part of the microcavity transmission spectrum as a function of time as deionized water and glucose solutions are flowed through the cavity array. The tilted geometry results in the presence of both longitudinal and transverse modes in the spectrum. The modes shift by around $11 \mathrm{~nm}$ on introduction of the glucose solution to the cavity; (b) Measured refractive index as a function of time as deionized water is replaced with glucose within the microcavity array via a mixture of flow and diffusion.

\subsection{Absorption-based sensing}


Absorption spectroscopy in principle provides a highly appealing approach to detection of a wide range of analytes, with no need for labelling. The technique offers universal detection capabilities, since every molecule absorbs light somewhere within the range of wavelengths accessible with standard laboratory light sources, as well as the possibility of single-wavelength, multiple-wavelength, or broadband detection depending on the number of analytes to be detected or discriminated against simultaneously. Unfortunately, the method tends to suffer from low sensitivity. Quantitatively, the fraction of light transmitted through an absorbing sample is given by the Beer-Lambert law:

$$
I=I_{0} \exp (-\alpha C \ell)
$$

where $I_{0}$ and $I$ are the light intensities incident on and transmitted through the sample, respectively, $\alpha$ is the absorption coefficient of the analyte, $C$ is its concentration, and $\ell$ is the (single pass) absorption path length, as defined previously in Section 2. When the absorption coefficient and/or the concentration of the analyte is low, the reduction in intensity of light passing through the sample becomes extremely small, and an optical absorption measurement becomes insensitive. Positioning the sample within an optical microcavity increases the effective path length by orders of magnitude, with a corresponding improvement in detection sensitivity.

Within a microcavity, optical absorption is detected via an attenuation of the intensity of cavity modes that overlap in wavelength with the absorption spectrum of the analyte. For a given cavity mode, Equation (3) may be used to relate the mode intensities transmitted through the cavity in the absence and presence of an absorbing sample to the concentration and absorption coefficient of the sample Rearranging Equation (3) yields

$$
\frac{1}{\alpha \ell}\left(\frac{I_{0}-I}{I}\right)=C L^{-1}
$$

If the absorption coefficient $\alpha$ and single-pass path length $\ell$ through the sample are known, and the transmitted intensity $I$ is measured for a number of sample concentrations, then the slope of a plot of the left hand side of Equation (7) against concentration yields the cavity enhancement factor, $L^{-1}$. A plot for a sample data set is shown in Figure 4(b).

The minimum concentration that can be detected corresponds to the minimum drop in transmitted intensity, $\Delta I_{\min }=\left(I_{0}-I\right)_{\min }$ that can be measured. Making the approximation that $I_{0}=I$ at this limit, Equation (3) yields

$$
C_{\min }=\frac{1}{\alpha \ell}\left(\frac{\Delta I_{\min }}{I_{0}}\right) L
$$

In general, $I_{0}$ is taken to be the intensity transmitted through a cavity filled with a 'blank' solution, e.g. solvent only (these traces are labelled 'No sample' in the data set shown in Figure $4(\mathrm{a})$ ), and $\Delta I_{\min }$ is taken to be three times the standard deviation over time of this baseline $I_{0}$ measurement.
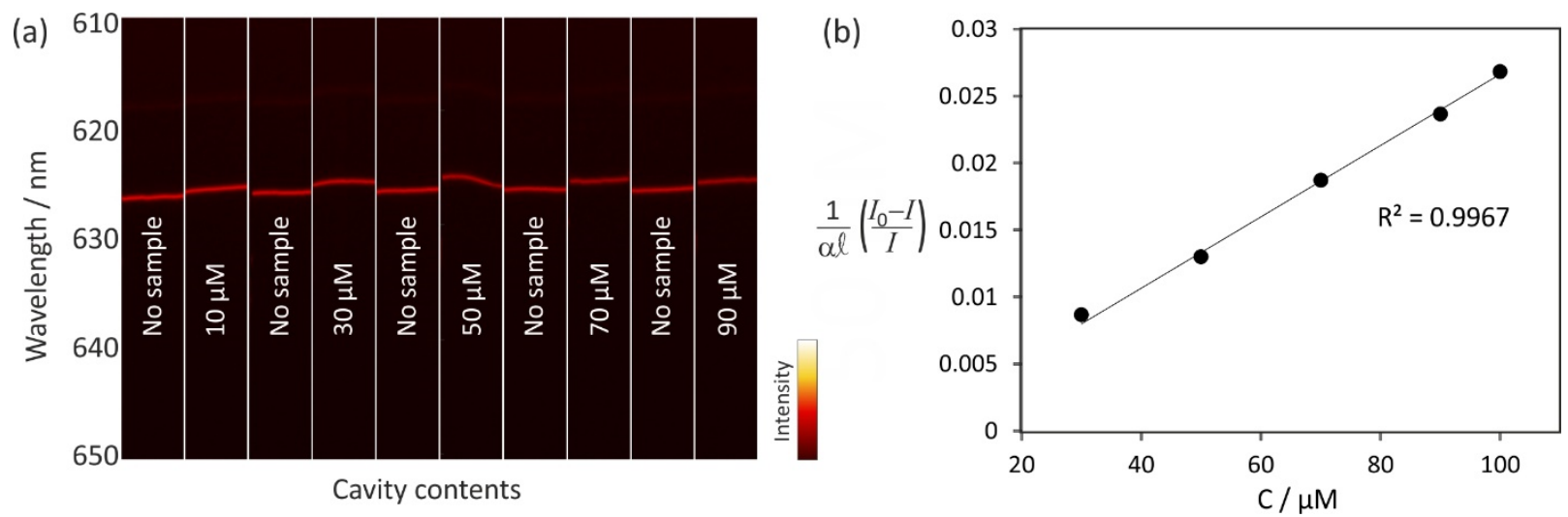
Figure 4: (a) Attenuation of a single cavity mode as increasing concentrations of methylene blue in ethanol are introduced into the microcavity; (b) Plot of Equation (7) used to determine the cavity enhancement factor. The data were fitted to a straight line passing through the origin with a regression coefficient of $R^{2}=0.9967$.

The data set in Figure 4 corresponds to a measurement in which increasing concentrations of methylene blue in ethanol were flowed through a microcavity. The experimental arrangement was similar to that employed for the refractive index sensing measurements described previously. In between methylene blue measurements, the microcavities were flushed with ethanol to check that $I_{0}$ returned to its baseline value. Figure 4(a) illustrates the attenuation of the cavity modes with increasing concentration of analyte, as well as a small shift in mode frequency due to the slightly differing refractive indices of the ethanol and analyte solutions. Figure 4(b) shows a plot of Equation 7. For this data set, the plot yields a cavity-enhancement factor of 266 and the minimum detectable concentration determined from Equation (8) is $25 \mu \mathrm{M}$. Given the $0.47 \mathrm{fL}$ mode volume, this corresponds to detection of around $1.18 \times 10^{-20} \mathrm{~mol}$, or 7000 molecules of methylene blue within the probed region. With improved mirror reflectivity, and use of a laser source in place of the quartz halogen lamp, we have recently improved this detection limit to less than 100 molecules ${ }^{22}$, and we hope to push the detection limit still further towards the 'holy grail' of recording the absorption spectrum of a single molecule.

\subsection{Particle trapping and tracking}

In Section 4.1 we demonstrated the high sensitivity of the cavity mode frequencies to the refractive index of the cavity contents. In that case, the refractive index of the entire liquid contents of the microcavity was altered through the addition of dissolved glucose. However, we have also shown that the increase in optical path length caused by diffusion of a nanoparticle through a cavity mode yields a large enough frequency shift to be detected ${ }^{23}$. The observed frequency shift in this case depends on the size of the particle and on the difference between the refractive indices of the nanoparticle and the solvent. Figure 5(a) shows the transient shifts in frequency of a longitudinal mode in a microcavity as a polystyrene nanosphere $300 \mathrm{~nm}$ in diameter diffuses through the mode volume. The two traces in the figure correspond to the same mode in two separate cavities: the upper trace is for a 'reference' cavity in which no particle is present. In the lower trace, the wavelength perturbations due to the particle are clearly observed. The time required for a particle of this size to diffuse across the diameter of a cavity mode is on the order of $0.1 \mathrm{~s}$, in good agreement with the duration of the observed frequency shifts in the cavity mode.

(a)

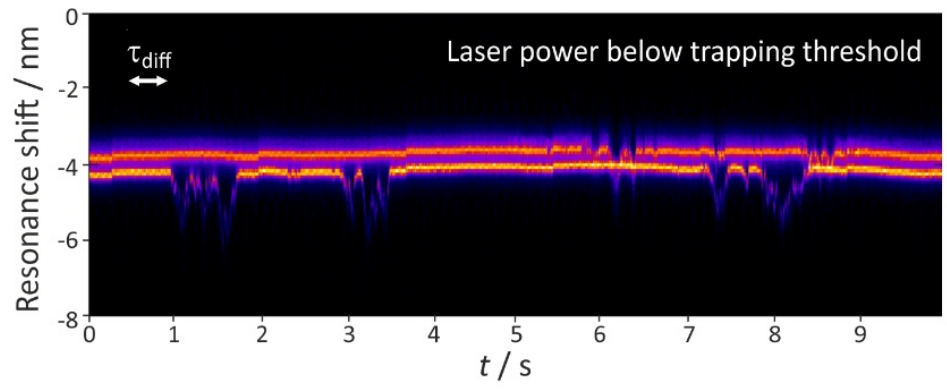

(b)

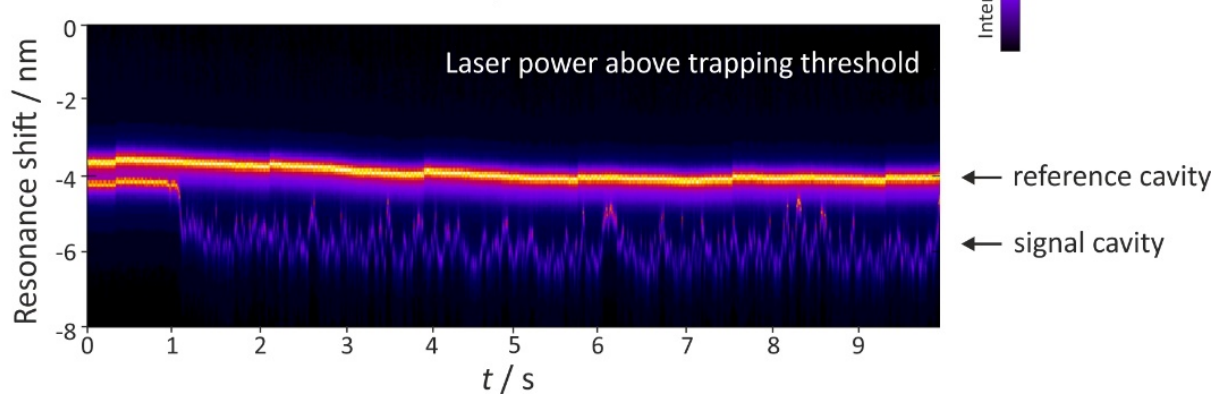

$\longleftarrow$ reference cavity

$\leftarrow$ signal cavity

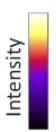

$\leftarrow$ reference cavity

signal cavity

Figure 5: (a) Fluctuations in cavity mode frequencies as $300 \mathrm{~nm}$ polystyrene nanospheres diffuse through a longitudinal cavity mode (the upper trace is for a reference cavity containing no particle, to account for mode noise common to both cavities); (b) trapping of a nanosphere within the cavity mode at higher illumination 
intensity. In both cases, the time required for the nanoparticle to diffuse across the diameter of the cavity is $-0.1 \mathrm{~s}$ Spectra were recorded by recording the laser transmission as the cavity length was scanned.

If the intensity of the excitation light is high enough, nanoparticles can be optically trapped within the cavity mode. Figure 5 (b) shows an example from the same experiment in which the illumination power has been increased to exceed the trapping threshold. In contrast with the example shown in Figure 5(a), in which the light intensity in the cavity modes is relatively low and the shifts in cavity mode frequency caused by the presence of the particle are transient, when trapping occurs the shift remains over timescales much longer than the particle diffusion time. Brownian motion of the trapped particle in the trapping field is apparent in the 'noise' on the resulting shifted resonance signal. The time autocorrelation function of the modeshift provides useful information about the diffusion of the particle through the confined mode, and can be analysed to determine the size and refractive index of the particle. Details of the required data analysis are given in reference 23 .

\section{Outlook}

While microcavity-based sensors relying on chemical binding to a resonator surface have been in existence for some time, chemical sensing in open-access microcavities is a relatively new enterprise. We have demonstrated that such microcavities provide a versatile platform for sensing applications, and are compatible with a number of different sensing modalities. To date, we have demonstrated refractive index sensing, absorption sensing, and particle tracking and trapping, but sensors based on cavity-enhanced fluorescence, FRET (fluorescence/Förster resonance energy transfer), or Raman scattering are also possible. There is considerable scope for further development of the sensing platform to improve detection sensitivity and ease of use. Planned improvements include optimising the flow cell design and cavity mirror reflectivity, extending the accessible wavelength range to different regions of the spectrum, and exploring the compatibility of microcavity measurements with alternative types of light source and detector. The full power of open access microcavities for enhanced chemical sensing will be revealed over the next few years, as these devices move from the proof of concept stage into testing for a range of real-world sensing applications.

\section{References}

\footnotetext{
${ }^{1}$ Cavity-enhanced spectroscopy and sensing, Edited by Hans-Peter Loock and Gianluca Gagliardi (Springer,2014).

${ }^{2}$ www.tigeroptics.com

${ }^{3}$ www.picarro.com

${ }^{4}$ C. M. Rushworth, Y. Yogarajah, Y.Zhao, H. Morgan, and C. Vallance, Sensitive analysis of trace water analytes using colourimetric cavity ringdown spectroscopy, Analytical Methods, 5, 239-247 (2013).

${ }^{5}$ S. R. T. Neil, C. M. Rushworth, C. Vallance, and S. R. Mackenzie, Broadband cavity-enhanced absorption spectroscopy for real-time, in situ spectral analysis of microfluidic droplets, Lab on a Chip, 11, 3953-3955 (2011).

${ }^{6}$ C. M. Rushworth, J. Davies, J. Cabral, P. Dolan, J. M. Smith, and C. Vallance, Cavity-enhanced optical methods for online microfluidic analysis, Chem. Phys. Lett., 554, 1 (2012).

${ }^{7}$ D. James. R. G. Oag, C. M. Rushworth, J. W. L. Lee, J. Davies, J. T. Cabral, and C. Vallance, High sensitivity online detection for microfluidics via cavity ringdown spectroscopy, RSC Advances, 2(12), 5376-5384 (2012).

${ }^{8}$ See for example M. R. Foreman, J. D. Swaim, and F. Vollmer, Whispering gallery mode sensors, Adv. Opt. Photonics, 7(2), 168-240 (2015), and references therein.

${ }^{9}$ K. J. Vahala, Optical Microcavities, Nature, 424, 839-846 (2003).

${ }^{10}$ P. R. Dolan, G. M. Hughes, F. Grazioso, B. R. Patton, and J. M. Smith, Femtoliter tunable optical cavity arrays, Optics Lett., 35(21), 3556-3558 (2010).

${ }^{11}$ Z. Y. Di, H. V Jones, P. R. Dolan, S. M. Fairclough, M. B. Wincott, J. Fill, G. M. Hughes, and J. M. Smith, Controlling the emission from semiconductor quantum dots using ultra-small tunable optical microcavities, New J. Phys., 14(10), 103048 (2012).
} 
${ }^{12}$ A. A. P. Trichet, P. R. Dolan, D. M. Coles, G. M. Hughes, and J. M. Smith, Topographic control of open-access microcavities at the nanometer scale, Optics Express, 23, 17205-17216, (2015).

13 M. Trupke, E. A. Hinds, S. Eriksson, E. A. Curtis,Z. Moktadir, E. Kukharenka, and M. Kraft, Microfabricated highfinesse optical cavity with open access and small volume, Appl. Phys. Lett., 87, 211106 (2005).

${ }^{14}$ T Steinmetz, Y. Colombe, D. Hunger, T. W. Hënsch, A Balocchi, R. J Warburton, and J. Reichel, Stable fiber-based Fabry-Perot cavity, Appl. Phys. Lett., 89, 111110 (2006).

${ }^{15}$ G. Cui, J. M. Hannigan, R. Loeckenhoff, F. M. Matinaga, M. G. Raymer, S. Bhongale, M. Holland, S. Mosor, S. Chatterjee, H. M. Gibbs, and G. Khitrova, A hemispherical high-solid-angle optical micro-cavity for cavity-QED studies, Opt. Express, 14, 2289-2299 (2006).

${ }^{16}$ A. Muller, E. B. Flagg, M. Metcalfe, J. Lawall, and G. S. Solomon, Coupling an epitaxial quantum dot to a fiber-based external-mirror microcavity, Appl. Phys. Lett., 95, 173101 (2009)

${ }^{17}$ A. A. P. Trichet, J. Foster, N. E. Omori, D. James, P. R. Dolan, G. M. Hughes, C. Vallance, and J. M. Smith, Open-access optical microcavities for lab-on-a-chip refractive index sensing, Lab on a Chip, 14(21), 4244-4249 (2014).

${ }_{18}$ M. Mader, J. Reichel, T. Hänsch and D. Hunger, A scanning cavity microscope, Nature Commun. 6, 7249 (2015)

${ }^{19}$ H. Kelkar, D. Wang, D. Martin-Cano, B. Hoffmann, S. Christiansen, S. Götzinger and V Sandoghdar, Sensing nanoparticles with a cantilever-based scannable optical cavity of low finesse and sub- $\lambda^{3}$ volume, Phys. Rev. Applied 4, 054010 (2015).

${ }^{20}$ S. E. Fiedler, A. Hese, and A. A. Ruth, Incoherent broad-band cavity-enhanced absorption spectroscopy, Chem. Phys. Lett., 371(3-4), 284, (2003).

${ }^{21}$ We have recently recorded a finesse of 30,000 in similar cavities, suggesting a limit of detection of around 17,000 molecules

${ }^{22}$ D. James, A. A. P. Trichet, C. Vallance, and J. M. Smith, Open-access optical microcavities for the analysis of submicrolitre liquid volumes using cavity enhanced absorption spectroscopy, in preparation.

${ }^{23}$ A. A. P. Trichet, P. R. Dolan, D. James, G. M. Hughes, C. Vallance, and J. M. Smith, Nanoparticle trapping and characterisation in an optical open-access microcavity, submitted February 2015. 